\title{
Interactions between procedural learning and cocaine exposure alter spontaneous and cortically evoked spike activity in the dorsal striatum
}

\author{
Janie M. Ondracek ${ }^{1 \dagger}{ }^{\text {, Ingo Willuhn }}{ }^{2 \dagger}$, Heinz Steiner $^{2}$ and Anthony R. West ${ }^{1 *}$ \\ 1 Department of Neuroscience, The Chicago Medical School/Rosalind Franklin University of Medicine and Science, North Chicago, IL, USA \\ 2 Department of Cellular and Molecular Pharmacology, The Chicago Medical School/Rosalind Franklin University of Medicine and Science, North Chicago, IL, USA
}

\section{Edited by:}

Steven L. Roberds, Pfizer, USA

Reviewed by:

David M. Lovinger, National Institutes

of Health, USA

Steven L. Roberds, Pfizer, USA

\section{*Correspondence:}

Anthony R. West, Department of Neuroscience, Rosalind Franklin

University of Medicine and Science, 3333 Green Bay Road, North Chicago,

IL 60064, USA.

e-mail: anthony.west@rosalindfranklin. edu

\section{${ }^{\dagger}$ Present address:}

Janie M. Ondracek, Institute of Neuroinformatics, University of Zurich and Eidgenössische Technische Hochschule Zurich, Zurich, Switzerland; Ingo Willuhn, Department of Psychiatry and Behavioral Sciences, University of Washington, Seattle, WA, USA.
We have previously shown that cocaine enhances gene regulation in the sensorimotor striatum associated with procedural learning in a running-wheel paradigm. Here we assessed whether cocaine produces enduring modifications of learning-related changes in striatal neuron activity, using single-unit recordings in anesthetized rats 1 day after the wheel training. Spontaneous and cortically evoked spike activity was compared between groups treated with cocaine or vehicle immediately prior to the running-wheel training or placement in a locked wheel (control conditions). We found that wheel training in vehicle-treated rats increased the average firing rate of spontaneously active neurons without changing the relative proportion of active to quiescent cells. In contrast, in rats trained under the influence of cocaine, the proportion of spontaneously firing to quiescent cells was significantly greater than in vehicle-treated, trained rats. However, this effect was associated with a lower average firing rate in these spontaneously active cells, suggesting that training under the influence of cocaine recruited additional low-firing cells. Measures of cortically evoked activity revealed a second interaction between cocaine treatment and wheel training, namely, a cocaine-induced decrease in spike onset latency in control rats (locked wheel). This facilitatory effect of cocaine was abolished when rats trained in the running wheel during cocaine action. These findings highlight important interactions between cocaine and procedural learning, which act to modify population firing activity and the responsiveness of striatal neurons to excitatory inputs. Moreover, these effects were found $24 \mathrm{~h}$ after the training and last drug exposure indicating that cocaine exposure during the learning phase triggers long-lasting changes in synaptic plasticity in the dorsal striatum. Such changes may contribute to the transition from recreational to habitual or compulsive drug taking behavior.

Keywords: corticostriatal transmission, dorsal striatum, medium-sized spiny neuron, habit formation, procedural learning, addiction

\section{INTRODUCTION}

Psychostimulant addiction entails compulsive drug taking. Motivated behavior is critically dependent on interactions between the cortex and the basal ganglia (Mogenson et al., 1980). According to current models, these interactions are mediated by parallel, functionally diverse circuits that interconnect the cortex and the basal ganglia (Alexander et al., 1986, 1990; Haber, 2008; DeLong and Wichmann, 2010). It has been proposed that addiction involves aberrant procedural learning produced by drug exposure, and that this effect is mediated by drug-induced neuroplasticity in cortico-basal ganglia circuits through the dorsal striatum (White, 1996; Robbins and Everitt, 1999; Berke and Hyman, 2000; Hyman and Malenka, 2001; Gerdeman et al., 2003; Everitt and Robbins, 2005).

Current models of basal ganglia function indicate that "motor" circuits involving the premotor and sensorimotor cortex and the related striatal sectors are active during the execution of patterned behavior. The phasic activation of striatal medium-sized spiny neurons (MSNs) that occurs during movement is believed to be enabled or triggered by robust activation of convergent cortical inputs (see Wilson, 2004, for review). Thus, MSNs in the sensorimotor striatum fire in a manner correlated with specific aspects of motor performance (West et al., 1990; Tschanz et al., 1991; Haracz et al., 1993; Carelli et al., 1997; Jog et al., 1999; Costa et al., 2004) and this activity seems to be modified during procedural learning (Carelli et al., 1997; Jog et al., 1999; Brasted and Wise, 2004; Costa et al., 2004). MSN activity is also altered by acute exposure to psychostimulants and other dopamine agonists (Tschanz et al., 1991; Haracz et al., 1993; Pederson et al., 1997; Rebec et al., 1997; West et al., 1997; White and Kalivas, 1998). Moreover, the interactions between afferent drive and intrinsic membrane properties of MSNs are potently modulated by dopamine (Nicola et al., 2000; Reynolds and Wickens, 2002; West et al., 2003; Surmeier et al., 2007) and are modified during motor learning and repeated exposure to psychostimulants (Centonze et al., 2002, 2006).

Long-term neuroplasticity that can last for a lifetime such as that underlying addiction is most likely mediated by altered gene regulation (Nestler, 2001). Indeed, psychostimulant-induced gene regulation is particularly prominent in MSNs of the striatum (Berke and Hyman, 2000; Steiner, 2010). In previous studies, we have mapped changes in striatal gene expression produced by cocaine to determine which corticostriatal circuits are affected by this drug (Willuhn et al., 2003; Unal et al., 2009). The focus was on 
transcription factors/immediate-early genes (IEGs; c-fos, zif 268), and postsynaptic density proteins (e.g., Homer la), as these regulate neuroplasticity by modifying the activity of effector genes and synaptic plasticity, respectively. Our results showed that sensorimotor striatal sectors that receive convergent inputs from the sensorimotor and the prefrontal/medial agranular cortex are most prone to cocaine-induced changes in expression of these genes (Willuhn et al., 2003; Unal et al., 2009; see Steiner, 2010, for review).

Given the proposed role of drug-induced alterations in procedural learning in addiction, as well as the previous finding that the above genes are involved in various forms of learning (see Steiner, 2010), we next investigated whether procedural learning was associated with changes in such gene regulation in the sensorimotor striatum and whether these effects were affected by cocaine. We employed a procedural learning task that is dependent on normal function of the sensorimotor striatum, that is, skill learning in a running wheel (Willuhn and Steiner, 2008, 2009). In these studies, we compared rats that trained on a running wheel under the influence of cocaine (or vehicle) with cocaine (or vehicle)-treated untrained controls. We observed that 1 day after the training, cocaine-treated, trained rats displayed a greater IEG response to a cocaine challenge in the sensorimotor striatum, compared with cocaine-treated, untrained controls (Willuhn et al., 2003; Willuhn and Steiner, 2006). This effect was restricted to the beginning of the training (Willuhn and Steiner, 2006), when rats learn to run on the wheel (Willuhn and Steiner, 2008), and was thus not related to running in the wheel per se (exercising). Similar but more modest training effects on gene regulation were seen when rats trained after vehicle administration (Willuhn and Steiner, 2005), suggesting that cocaine abnormally enhances learning-associated gene regulation. These findings suggested that such procedural learning is associated with synaptic plasticity in the sensorimotor striatum, and that these processes are altered by cocaine.

In the current study, we utilized in vivo recording techniques to determine whether procedural learning in the running-wheel model (Willuhn and Steiner, 2006, 2008) is associated with electrophysiological changes in striatal MSNs that receive inputs from the frontal cortex, and whether these effects are modified by cocaine.

\section{MATERIALS AND METHODS SUBJECTS}

Male Sprague-Dawley rats (Harlan, Madison, WI, USA) weighing $180-220 \mathrm{~g}$ at the beginning of the experiments were housed two per cage under standard laboratory conditions $\left(21-23^{\circ} \mathrm{C}\right)$ and maintained on a 12:12 h light/dark cycle with food and water available ad libitum. All animal protocols were approved by the Rosalind Franklin University of Medicine and Science Institutional Animal Care and Use Committee and adhered to the Guide for the Care and Use of Laboratory Animals published by the USPHS.

\section{DRUG TREATMENT AND WHEEL TRAINING}

Our previous studies showed that rats trained to run on a wheel under the influence of cocaine exhibited a more robust increase in striatal c-fos expression in response to a cocaine challenge given 1 day after the training, compared with cocaine-treated untrained (locked wheel) controls (Willuhn and Steiner, 2006). This enhanced c-fos response peaked after the second day of training and disappeared by the eighth day of training, indicating that this effect was temporally associated with the learning phase (Willuhn and Steiner, 2006). Thus, in the current study, a 2-day training protocol was employed, and electrophysiological recordings were performed 1 day after the training. On two consecutive days, rats received an injection of cocaine hydrochloride ( $25 \mathrm{mg} / \mathrm{kg}$, Sigma, St. Louis, MO, USA; in $0.02 \%$ ascorbic acid, i.p.) or vehicle. Immediately after the injection, they were placed on either a running wheel ("trained") or an immobilized wheel ("locked") for a 60-min training session. Animals that are allowed to run learn to control/balance the wheel in order to stay at the bottom of the wheel during running (Willuhn and Steiner, 2006, 2008). This response was tested (see Willuhn and Steiner, 2008) 1 day after the training, and the few animals that did not learn were excluded.

\section{SURGERY}

Prior to surgery, animals were deeply anesthetized with chloral hydrate ( $400 \mathrm{mg} / \mathrm{kg}$; Sigma) and placed in a stereotaxic apparatus as described previously (Ondracek et al., 2008). Burr holes ( 2-3 mm in diameter) were drilled in the skull overlying the right frontal cortex and dorsal striatum. The dura mater was resected, and the stimulating and recording electrodes were lowered into the brain using a Narishige or Kopf micromanipulator. The level of anesthesia was periodically verified via the hind limb compression reflex and maintained using supplemental administration of chloral hydrate as previously described (West and Grace, 2004; Sammut et al., 2006). Temperature was monitored using a rectal probe and maintained at $37^{\circ} \mathrm{C}$ using a heating pad (Vl-20F, Fintronics Inc., Orange, CT, USA).

\section{ELECTRICAL STIMULATION}

In our previous studies, cocaine-enhanced, wheel learningassociated IEG induction was preferentially seen in parts of the sensorimotor striatum that also receive inputs from the prefrontal/ medial agranular cortex (Willuhn et al., 2003; Willuhn and Steiner, 2006), suggesting a role for such prefrontal input in learning-associated neuroplasticity in the striatum. In order to identify striatal MSNs under the influence of prefrontal/medial agranular input, a twisted-pair bipolar stimulating electrode (Plastics One, Roanoke, VA, USA) was implanted into the frontal cortex, targeting the medial agranular area ipsilateral to the striatal recording pipette. Electrical stimuli with a duration of $0.2-0.5 \mathrm{~ms}$ and an intensity of $0.25-1.25 \mathrm{~mA}$ were generated using a stimulator and photoelectric constant current/stimulus isolation unit (S88 stimulator with PSIU6F stimulus isolation unit, Grass Instruments, Quincy, MA, USA) and delivered in single pulses $(0.5 \mathrm{~Hz})$ for a duration of 50-100 s as previously described (Ondracek et al., 2008).

\section{EXTRACELLULAR RECORDINGS}

All electrophysiological recordings were performed 1 day after the final cocaine administration/wheel session. A between-subjects approach was used throughout, and the experimenter was blind with respect to the group identity during the recording session.

Extracellular recording microelectrodes were manufactured from $2.0 \mathrm{~mm}$ o.d. borosilicate glass capillary tubing (WPI, New York, NY, USA) using a vertical micropipette puller (model PE-21, Narishige) as previously described (West and Grace, 2000; Ondracek et al., 2008). 
Briefly, electrodes were broken back against a glass rod under microscopic control to an approximately $1 \mu \mathrm{m}$ tip diameter and filled with $2 \mathrm{M} \mathrm{NaCl}$. Microelectrode resistance was measured in vivo at $1000 \mathrm{~Hz}$ with a current of $10 \mathrm{nA}$ using an FHC Xcell 3+ microelectrode amplifier/impedance check [Frederick Haer Company (FHC), Bowdoinham, ME, USA] and ranged from 10 to $15 \mathrm{M} \Omega$.

In order to isolate single-units that received input from the frontal cortex, extracellular microelectrodes were lowered incrementally through the dorsocentral and dorsolateral (sensorimotor) striatum that displayed wheel learning-associated gene regulation (Willuhn et al., 2003; Willuhn and Steiner, 2005, 2006), using a micromanipulator (MO-8, Narishige), while single pulse electrical stimuli (see above) were administered to the cortex (Ondracek et al., 2008). Once a cell was detected, the position of the recording electrode was adjusted to maximize the spike signal to background noise ratio $(\geq 4: 1)$. In a subgroup of neurons/animals, stimulation currents were titrated to an intensity (range, 200-1210 $\mu \mathrm{A}$ ) that reliably evoked spike activity approximately $50 \%$ of the time (e.g., $\sim 50$ spikes in response to 100 stimulus pulses delivered at $0.5 \mathrm{~Hz}$ ) to enable comparisons of cortically evoked activity across groups. The spontaneous (no stimulation) activity of striatal neurons was then recorded for approximately $5 \mathrm{~min}$. The response to cortical stimulation was reaffirmed before searching for the next cell. Typically, two to four cells were recorded in as many tracks per animal. Extracellular electrode potentials were acquired and analyzed as previously described (Ondracek et al., 2008; Threlfell et al., 2009; Sammut et al., 2010). Striatal neurons exhibiting spike characteristics that could be described as tonic (i.e., cholinergic or nitrergic interneurons) or fast-spiking (GABAergic interneurons which respond to low intensity cortical stimulation with a high-frequency train of short duration ( $<0.9 \mathrm{~ms}$ ) action potentials) (Wilson, 2004; Mallet et al., 2005) were not included. Therefore, it is highly likely that the vast majority or all of the single-units recorded in this study were MSNs.

\section{DATA ANALYSIS AND STATISTICS}

Peri-stimulus time histograms were constructed ( $1.0 \mathrm{~ms}$ bins) for each cortical stimulation trial and spike probabilities were calculated by dividing the number of evoked action potentials (either 0 or 1 per pulse) by the number of stimuli delivered (Ondracek et al., 2008). Single-unit and group data were also summarized using spike latency and standard deviation (SD) of latency plots as indicated. Firing rate histograms were constructed $(1.0 \mathrm{~ms}$ bins of 2 min epochs) from cells isolated using single-pulse stimulation of the frontal cortex (Ondracek et al., 2008). Neurons were considered "spontaneously active" if they fired multiple action potentials $(>1)$ during a 2 -min recording period. The statistical significance of drug-induced changes in spike activity was determined by using either a Chi-square test or two-way analysis of variance (ANOVA; Sigma Stat, Jandel). A Tukey post hoc test was used to determine which group (s) contributed to overall differences seen with ANOVA.

\section{HISTOLOGY}

After completion of each experiment, the rat was deeply anesthetized and perfused transcardially with ice-cold saline followed by $10 \%$ formalin in buffered phosphate (PB; EMS, Hatfield, PA, USA).
Brains were removed and postfixed in formalin/sucrose solution (30\%) and stored at $4^{\circ} \mathrm{C}$ until saturated. Brains were then sectioned into $50 \mu \mathrm{m}$ coronal slices, mounted, and stained with a Neutral Red/ Cresyl Violet (10:1) solution to allow for histological assessment of stimulating and recording electrode tracks (Threlfell et al., 2009).

\section{RESULTS}

\section{STIMULATING AND RECORDING ELECTRODE PLACEMENTS}

All identified stimulating electrode tips were confirmed to lie in the frontal cortex between 2.7 and $4.7 \mathrm{~mm}$ anterior to bregma, 1.5 and $3.4 \mathrm{~mm}$ lateral to the midline, and 1.5 and $4.2 \mathrm{~mm}$ ventral to the surface of the brain (Paxinos and Watson, 1998). Identified placements for extracellular recording electrodes implanted into the striatum were verified to lie between 0.20 and $1.7 \mathrm{~mm}$ anterior to bregma, 1.3 and $3.8 \mathrm{~mm}$ lateral to the midline, and 3.3 and $6.9 \mathrm{~mm}$ ventral to the brain surface (Paxinos and Watson, 1998).

\section{EFFECTS OF WHEEL TRAINING ON SPONTANEOUS ACTIVITY IN THE STRIATUM}

Examples of spontaneous firing activity in striatal neurons are shown in Figures 1A,B. Comparisons of the proportion of spontaneously firing vs. quiescent striatal cells across all groups revealed an overall effect of running-wheel training and cocaine on population activity (Table 1; Chi-square $=9.759$ with df $3, p<0.05$ ). Additionally, 1 day after the 2-day training, the proportion of spontaneously firing striatal cells receiving synaptic inputs from the frontal cortex was significantly greater in animals trained under the influence of cocaine as compared to vehicle-treated, trained controls (Table 1; Chi-square $=7.405$ with $\mathrm{df} 1, p<0.01$ ). The proportion of spontaneously active cells in animals confined to the locked wheel did not differ between cocaine- and vehicle-treated groups $(p>0.05)$. Also, no significant differences were observed within (trained vs. locked) vehicle- or cocaine-treated groups (Table $1 ; p>0.05$ ).

Analysis of overall group spike activity showed that the firing rate of all 251 neurons (active and quiescent) studied was not significantly affected by running-wheel training and/or cocaine treatment (vehicle locked, mean \pm SEM, $0.21 \pm 0.09 \mathrm{~Hz}, n=51$ cells $/ 17$ rats; vehicle trained, $0.19 \pm 0.07, n=56$ cells $/ 20$ rats; cocaine locked, $0.17 \pm 0.06, n=84$ cells $/ 21$ rats; cocaine trained, $0.11 \pm 0.03$, $n=60$ cells $/ 20$ rats; $p>0.05$; two-way ANOVA). This was not unexpected given that previous studies of putative striatal MSNs that used similar approaches have consistently found that these neurons exhibit a broad range of spontaneous firing rates (Mallet et al., 2005; Ondracek et al., 2008). Examination of the overall firing rate distribution revealed that the majority of the variance in the data set resulted from quiescent cells (i.e., firing rate $=0$ ) and a minority of outliers (nine cells), present in all groups, that fired at much higher frequencies ( $>2$ SDs) than the group means (Figure 1C). In order to allow meaningful comparisons of firing activity across groups, the analysis was therefore limited to spontaneously active neurons (excluding quiescent cells) that exhibited firing rates not $>2$ SDs above the overall group mean (Figure 1C). Comparisons with this data set revealed a significant main effect of cocaine treatment (cocaine vs. vehicle) on firing rates [Figure 1D; $F(1,87)=6.913, p=0.01$, two-way ANOVA]. There was also a robust trend for a main effect of wheel training (trained vs. locked) $[F(1,87)=3.419, p=0.068]$, as well as a significant interaction 
between drug treatment and training $[F(1,87)=6.265, p=0.014]$. Post hoc analysis showed that spontaneously active cells recorded in animals that trained without cocaine (i.e., after a vehicle injection)
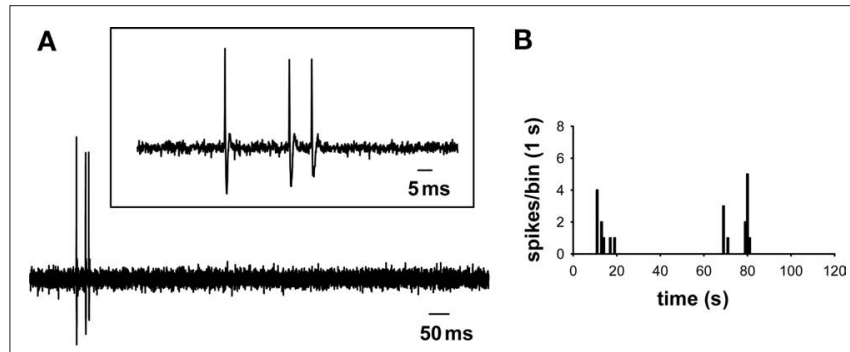

C

D
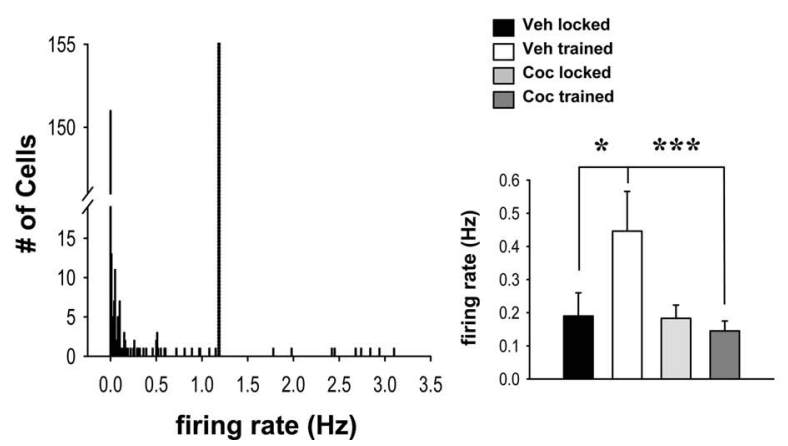

FIGURE 1 | Cocaine prevents wheel training-associated increases in population firing in spontaneously active striatal neurons that receive input from the frontal cortex. (A) Representative recording of spontaneous activity of a striatal neuron identified by electrical stimulation of the frontal cortex. Inset: Magnification of the spontaneous spike activity shown below. (B) Representative firing rate histogram (2 min epoch) depicts the spontaneous activity of the single-unit shown in (A) (note the slow and irregular firing activity characteristic of striatal MSNs). (C) The firing rate distribution for all cells recorded in this study shows that most cells were quiescent (151/251) or fired at relatively low rates (91/251). A small group of outliers (9/251), made up of cells from all treatment groups, exhibited a considerably higher firing rate and was thus excluded from the analysis (the cut-off, indicated by dashed line, was set at 2 SDs above group mean). (D) Comparisons of firing rates (mean \pm SEM) of spontaneously active neurons across groups ( $n=15-36$ cells per group) revealed a facilitatory effect of running-wheel training (after vehicle administration) on spontaneous firing $\left({ }^{*} p<0.05\right.$, two-way ANOVA, Tukey post hoc test). This effect was prevented in rats that trained under the influence of cocaine (vs. vehicle trained, ${ }^{* *} p<0.001$ )

Table 1 | Proportion of spontaneously firing striatal neurons in trained and untrained (locked) groups treated with vehicle or cocaine.

\begin{tabular}{lll}
\hline Treatment & Firing (>1 spike/120 s) & Quiescent \\
\hline VL & $33.3 \%(17 / 51$ cells $)$ & $66.7 \%(34 / 51$ cells $)$ \\
VT & $26.8 \%(15 / 56$ cells $)$ & $73.2 \%(41 / 56$ cells $)$ \\
CL & $42.9 \%(36 / 84$ cells $)$ & $57.1 \%(48 / 84$ cells $)$ \\
CT & $53.3 \%(32 / 60 \text { cells })^{*}$ & $46.7 \%\left(28 / 60\right.$ cells ${ }^{*}$
\end{tabular}

Ratios in parentheses indicate the number of cells exhibiting spontaneous activity or no activity (quiescent) per number of cells tested.

* Significantly different from VT control group as determined using a Chi-square test $(p=0.007)$. Data were derived from $n=17-21$ rats per group.

$V L$, vehicle locked; $V T$, vehicle trained; $C L$, cocaine locked; $C T$, cocaine trained. displayed, 1 day later, a significantly increased firing rate compared with vehicle-treated, untrained (locked) animals (Figure 1D; $p=0.011$, Tukey test). This effect was completely prevented by cocaine. Thus, in rats that trained under the influence of cocaine, cells showed a significantly lower firing rate than those in vehicletreated, trained controls $(p<0.001)$, and there was no significant difference between cells in cocaine-treated, trained animals and those in cocaine-treated, untrained controls $(p>0.05)$. There was also no difference in the firing rate of spontaneously active cells between cocaine-treated and vehicle-treated groups confined to the locked wheel (untrained controls) $(p>0.05)$.

\section{EFFECTS OF WHEEL TRAINING ON CORTICALLY EVOKED ACTIVITY IN STRIATAL NEURONS}

As described above, single-units were isolated using low-frequency electrical stimulation of the frontal cortex (Figure 2A). No significant differences in the minimal stimulating current needed to

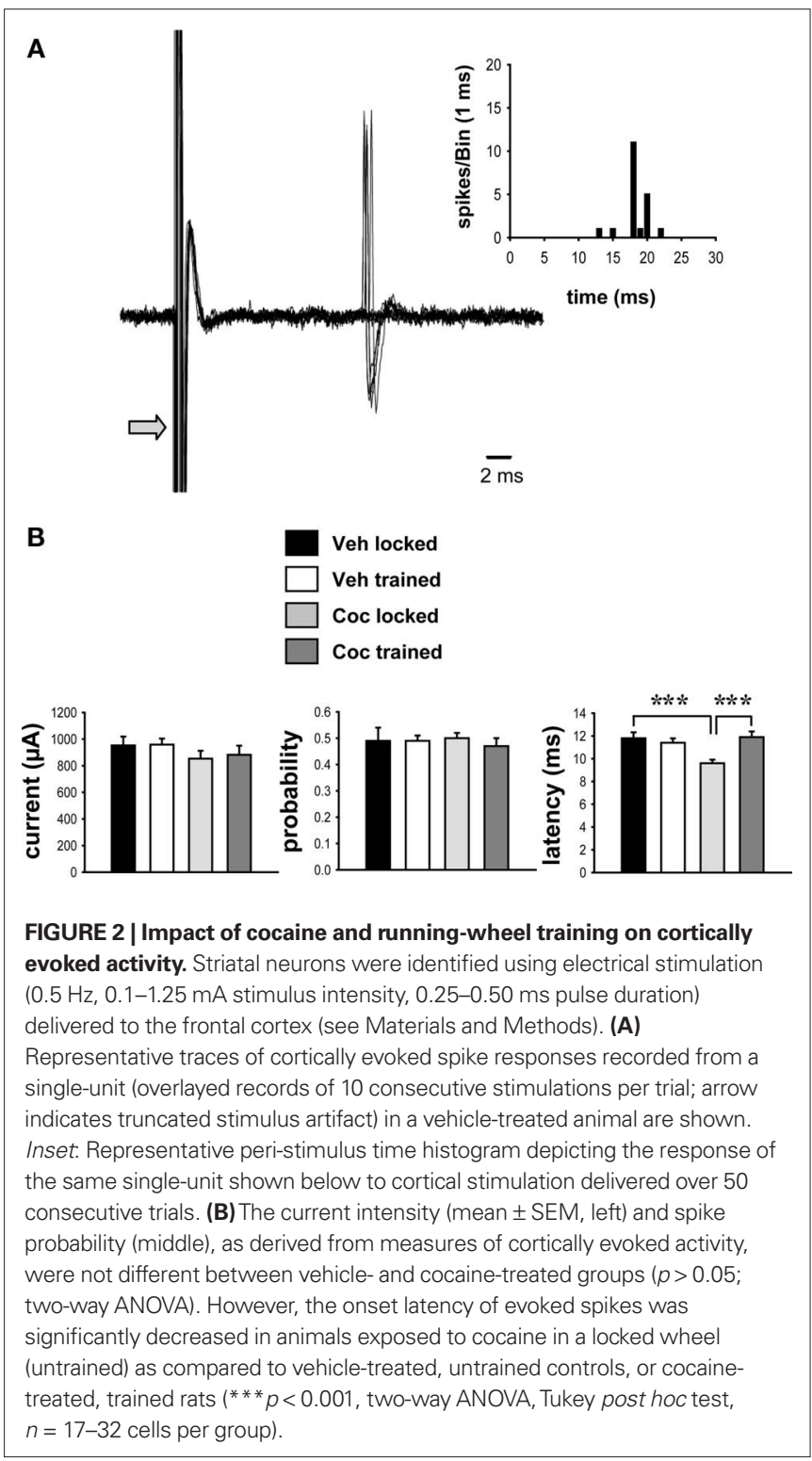


reach a response probability of approximately $50 \%$ were observed between these groups (Figure 2B; $p>0.05$, two-way ANOVA). The probability of evoking spikes during cortical stimulation was also not different across groups (Figure 2B; $p>0.05$ ). Comparisons of the onset latency of cortically evoked spikes across groups, however, showed a significant main effect of training [Figure 2B; $F(1,95)=4.774, p<0.05$, two-way ANOVA] and a strong trend toward a main effect of cocaine treatment $[F(1,95)=3.848$, $p=0.053]$. There was also a significant interaction between cocaine treatment and training $[F(1,95)=9.814, p<0.005]$. Post hoc comparisons revealed no effect of the wheel training on the spike onset latency in vehicle-treated rats $(p>0.05)$. However, cocaine treatment produced a significant decrease in the onset latency in rats confined to the locked wheel (untrained group) as compared to the vehicle-treated, untrained group (Figure 2B; $p<0.001$, Tukey test). This effect was prevented by the wheel training, as the cocaine-treated, trained group showed a significantly longer spike onset latency than the cocaine-treated, untrained group $(p<0.001)$ and did not differ from the vehicle-treated, trained group $(p>0.05)$. No significant effect of drug treatment or wheel training was observed for the standard deviation of spike latency (vehicle locked, $1.44 \pm 0.18$; vehicle trained, $1.14 \pm 0.12$; cocaine locked, $1.23 \pm 0.10$; cocaine trained, $1.26 \pm 0.15 ; p>0.05$, twoway ANOVA).

\section{DISCUSSION}

This study examined whether procedural learning is associated with enduring alterations in spontaneous firing and cortically driven activity in MSNs of the striatum, with a focus on striatal MSNs that receive frontal cortical input, and whether cocaine modifies these changes. We used skill learning in the running wheel as a simple model for procedural learning and found that this learning (without cocaine) was associated with an increase in spontaneous firing activity in these striatal MSNs. Our results revealed several effects of cocaine on wheel training-associated changes in cell firing that persisted for at least $24 \mathrm{~h}$ after the training sessions. For example, animals that learned to run on the wheel under the influence of cocaine exhibited, 1 day later, a higher proportion of spontaneously firing cells that received frontal cortical input. However, this effect was associated with a decrease in the average firing rate in these cells. These findings suggest that cocaine exposure during this procedural training produces a persistent reorganization of synaptic transmission in striatal networks. In further support of this conclusion, we found that cocaine exposure produced an increase in responsiveness to frontal cortical input (expressed as a decrease in onset latency of cortically evoked spikes) in untrained controls and that this effect was abolished in animals that trained on the wheel under the influence of cocaine. These findings suggest that long-term facilitatory effects of cocaine on corticostriatal transmission may be depotentiated by neuroplastic changes occurring during motor learning.

\section{PROCEDURAL LEARNING IN THE RUNNING WHEEL IS ASSOCIATED WITH} ENHANCED NEURONAL ACTIVITY IN STRIATAL NETWORKS

The basal ganglia play an important role in learning and memory functions related to motor behavior (e.g., Graybiel et al., 1994; White, 1997; Packard and Knowlton, 2002; Schultz et al., 2003;
Kelley, 2004). Consistent with earlier findings in other procedural learning tasks (e.g., Jog et al., 1999; Brasted and Wise, 2004; Costa et al., 2004), we found that cell firing in the sensorimotor striatum changes in association with procedural learning in a runningwheel paradigm. What is learned in this task? Video analysis of training sessions revealed that, at the beginning of the training, rats are unable to run with an appropriate speed to remain at the bottom of the wheel. The rat is often either too fast or too slow relative to the speed of the wheel, which causes the wheel to swing (Willuhn and Steiner, 2006, 2008). Early in the training, the rat learns to adapt running and balance the moving wheel in order to avoid such swinging; this response can be tested before and after the training to determine to degree of learning (Willuhn and Steiner, 2008). Our previous studies demonstrated that this wheelskill memory lasts for weeks to months (Willuhn and Steiner, 2008, 2009), and that such memory consolidation is dependent on processes in the sensorimotor striatum (Willuhn and Steiner, 2008) that continue after the training session (Willuhn and Steiner, 2009). Given the persistent nature of this procedural memory and the role of the striatum, we hypothesized that such running-wheel learning involves a cascade of neuronal events, including early transiently enhanced activity in corticostriatal circuits, followed by molecular changes in MSNs that enable synapse modifications/ restructuring leading to enduring changes in network activity and long-term memory consolidation (see Willuhn and Steiner, 2006, 2009).

The current study investigated early electrophysiological correlates of this wheel learning, namely, possible changes in spontaneous and evoked activity in MSNs that persisted for at least 1 day after the training and thus could reflect an early step in the process of consolidation. Our results show that, in vehicletreated animals that learned to run on the wheel, spontaneously firing MSNs exhibited a $>2$-fold increase in average firing rate (as compared to untrained, vehicle-treated controls), while the proportion of spontaneously firing to quiescent cells remained unchanged. These findings suggest that the consolidation of longterm procedural memory involves an up-regulation of neuronal network activity in sensorimotor regions of the dorsal striatum. Our findings obtained in anesthetized rats 1 day after the training are consistent with recent observations from studies of awake mice. Costa et al. (2004) demonstrated that skill learning on an accelerated rotarod is primarily associated with increased firing of single-units in the sensorimotor striatum during running trials. These authors concluded that a rapid and extensive recruitment of task-related neurons occurs in cortex and striatum during early sessions of skill learning. Our study shows that such up-regulated firing persists for at least 1 day, even under anesthetized conditions which promote increased inhibitory tone in corticostriatal networks. Such network adaptations may represent a primary means by which motor performance is improved during early exposure to novel motor tasks (Costa et al., 2004). Moreover, this enhanced neuronal activity may facilitate (calcium-dependent) gene regulation (Willuhn and Steiner, 2006) necessary for longterm consolidation of the learned motor response (Willuhn and Steiner, 2009). Future studies will have to characterize the mechanisms underlying the observed increase in firing activity in these studies. 


\section{COCAINE DISRUPTS THE FACILITATORY EFFECTS OF WHEEL TRAINING ON SPONTANEOUS FIRING ACTIVITY}

Given the involvement of dopamine receptor signaling in procedural learning (e.g., Beninger and Miller, 1998; Reynolds et al., 2001), it is to be expected that psychostimulants affect such learning. Current thinking proposes that the compulsive nature of drug taking in addiction may be related to aberrant procedural learning produced by psychostimulant exposure, and consequently, excessive stimulation of dopamine receptors, and that this effect is mediated at least in part by drug-induced neuroplasticity in the sensorimotor striatum (Berke and Hyman, 2000; Gerdeman et al., 2003; Everitt and Robbins, 2005). Our previous studies showed that cocaine indeed modifies procedural learning in the running wheel via D1 dopamine receptor overstimulation. Thus, cocaine administered before the wheel training tends to impair wheel learning (Willuhn and Steiner, 2008), and this impairment can be reversed by appropriate D1 receptor blockade (Willuhn and Steiner, 2008).

The current study thus also investigated the possibility that cocaine modified learning-related neuroplastic changes in striatal firing activity. Our analysis of spontaneously active vs. quiescent neurons with inputs from the frontal cortex (largely medial agranular region) showed that the rats that trained on the wheel under the influence of cocaine displayed, 1 day later, a higher proportion of spontaneously active cells than vehicle-treated, trained rats. However, we also found that, on average, these cells had a significantly lower firing rate than spontaneously active cells in vehicle-treated, trained controls. Thus, cocaine exposure during the wheel training produced an enduring increase in the number of spontaneously firing striatal neurons, but these displayed a lower overall population firing rate compared with cells recorded after wheel training without cocaine. A higher proportion of spontaneously active MSNs is consistent with our previous finding of enhanced IEG inducibility in MSNs, as determined by gene expression area measures, after a 2-day running-wheel training (Willuhn et al., 2003; Willuhn and Steiner, 2005, 2006).

The functional consequences of this recruitment of low-firing cells by cocaine are unclear, but it can be speculated that the net effect may be a decrease in the signal-to-noise ratio in striatal networks. Thus, more cells firing at low levels may create background noise that could act to obscure phasic input signals. Phasic activation of MSNs occurs during purposeful movement or learned behavior and is believed to be driven by cortical inputs (see Introduction). Obscured cortical action commands would be expected to result in impaired adaptations of motor responses, as we indeed have observed in the wheel tests after cocaine exposure during the training (Willuhn and Steiner, 2008). Moreover, strengthened consolidation by cocaine (Willuhn and Steiner, 2009) of such impaired motor adaptations due to abnormally enhanced gene regulation (Willuhn and Steiner, 2006) may contribute to aberrant procedural learning in addiction.

\section{WHEEL TRAINING PREVENTS FACILITATORY EFFECTS OF COCAINE ON CORTICOSTRIATAL TRANSMISSION}

In the present study, all striatal neurons were isolated using cortical stimulation as previously described (Ondracek et al., 2008; Threlfell et al., 2009; Sammut et al., 2010). This approach also allowed us to examine the impact of wheel training and cocaine exposure on cortically evoked spike activity. Our results showed that neither cocaine nor training affected the probability of triggering a striatal response $24 \mathrm{~h}$ after cocaine/training. However, cocaine exposure alone (i.e., in untrained locked wheel controls) produced a longlasting decrease in the onset latency of cortically evoked spikes. In contrast, if cocaine exposure occurred during the training on the wheel, then this decrease was prevented.

The observed decrease in onset latency suggests that the 2-day cocaine treatment increased the responsiveness of striatal neurons to excitatory corticostriatal input. This finding is perhaps not unexpected, given reports that a single cocaine injection can induce LTP in other neurons that lasts for up to 5 days post exposure (Ungless et al., 2001). Furthermore, neurons in the nucleus accumbens of cocaine-experienced rodents were found to exhibit both increases in surface levels and surface/intracellular ratios of the GluR1 and GluR2 AMPA receptor subunits (Boudreau and Wolf, 2005; Boudreau et al., 2007; Conrad et al., 2008), as well as potentiation of AMPA receptor-mediated synaptic transmission (Kourrich et al., 2007; Conrad et al., 2008), although these effects were typically seen after longer cocaine exposure. Increased corticostriatal synaptic efficacy in striatal neurons lasting longer than $24 \mathrm{~h}$ after repeated cocaine administration is also supported by recent studies by Calabresi and colleagues. This work shows that repeated cocaine treatment blocks the reversal of LTP at corticostriatal synapses, resulting in a persistent strengthening of glutamatergic transmission (Centonze et al., 2006).

Surprisingly, the facilitatory effect of cocaine on the responsiveness of striatal neurons to excitatory corticostriatal input was prevented in rats that trained on the wheel under the influence of this drug. As indicated above, there is evidence that procedural learning in the striatum may involve LTP- or LTD-like processes at corticostriatal synapses (Gerdeman et al., 2003; Mahon et al., 2004; Calabresi et al., 2007). In striatal slices, once established, LTP can be reversed to pre-stimulation (control) levels using a low-frequency stimulation protocol. This effect of low-frequency stimulation of cortical inputs is referred to as "synaptic depotentiation" (Picconi et al., 2003). The exact mechanisms underlying these altered cellular responses are not known, but intracellular (D1 receptor-PKA-DARPP32-PP1 pathways) and/or systems-level neuroadaptations may be involved (Calabresi et al., 2007). While speculative, the repeated activation of corticostriatal inputs occurring during running on the wheel may prevent or depotentiate the LTP-like effect observed in animals exposed to cocaine in the locked wheel. Further studies in reduced preparations will be necessary to clarify this issue.

\section{CONCLUSION}

It remains unclear precisely how cocaine modifies the neural circuits involved in dorsal striatal learning processes and whether/ how these alterations contribute to addiction. Nonetheless, the current findings demonstrate that the enduring effects of cocaine are associated with increases in background noise (increased proportion of low-firing, spontaneously active neurons), which may obscure the up-regulation of firing activity normally driven by experience-dependent neuroplasticity (i.e., the apparent cocaineinduced block of learning-dependent increases in population 
firing). These observations support the hypothesis that modification of procedural learning induced by cocaine exposure during running-wheel training is associated with a persistent reorganization of neuronal excitability in MSN networks. Furthermore, the observation that running-wheel training appeared to prevent the cocaine-induced facilitation of cortically evoked activity observed in non-running controls supports previous work suggesting that motor memory formation may involve depotentiation of synaptic efficacy in some corticostriatal circuits (Centonze et al., 2006). Together, these observations suggest that learning associated with

\section{REFERENCES}

Alexander, G. E., Crutcher, M. D., and DeLong, M. R. (1990). Basal gangliathalamocortical circuits: parallel substrates for motor, oculomotor, "prefrontal" and "limbic" functions. Prog. Brain Res. 85, 119-146.

Alexander, G. E., DeLong, M. R., and Strick, P. L. (1986). Parallel organization of functionally segregated circuits linking basal ganglia and cortex. Annu. Rev. Neurosci. 9, 357-381.

Beninger, R. J., and Miller, R. (1998). Dopamine D1-like receptors and reward-related incentive learning. Neurosci. Biobehav. Rev. 22, 335-345.

Berke, J. D., and Hyman, S. E. (2000). Addiction, dopamine, and the molecular mechanisms of memory. Neuron 25, 515-532.

Boudreau, A. C., Reimers, J. M., Milovanovic, M., and Wolf, M. E. (2007). Cell surface AMPA receptors in the rat nucleus accumbens increase during cocaine withdrawal but internalize after cocaine challenge in association with altered activation of mitogen-activated protein kinases. J. Neurosci. 27, 10621-10635.

Boudreau, A. C., and Wolf, M. E. (2005). Behavioral sensitization to cocaine is associated with increased AMPA receptor surface expression in the nucleus accumbens. J. Neurosci. 25, 9144-9151.

Brasted, P. J., and Wise, S. P. (2004). Comparison of learning-related neuronal activity in the dorsal premotor cortex and striatum. Eur. J. Neurosci. 19, 721-740.

Calabresi, P., Picconi, B., Tozzi, A., and Di Filippo, M. (2007). Dopaminemediated regulation of corticostriatal synaptic plasticity. Trends Neurosci. 30, 211-219.

Carelli, R. M., Wolske, M., and West, M. O. (1997). Loss of lever press-related firing of rat striatal forelimb neurons after repeated sessions in a lever pressing task. J. Neurosci. 17, 1804-1814.

Centonze, D., Costa, C., Rossi, S., Prosperetti, C., Pisani, A., Usiello, A., Bernardi, G., Mercuri, N. B., and Calabresi, P. (2006). Chronic cocaine prevents depotentiation at corticostriatal synapses. Biol. Psychiatry 60, 436-443.

Centonze, D., Picconi, B., Baunez, C., Borrelli, E., Pisani, A., Bernardi, G., and Calabresi, P. (2002). Cocaine and amphetamine depress striatal GABAergic synaptic transmission through D2 dopamine receptors. Neuropsychopharmacology 26, 164-175.

Conrad, K. L., Tseng, K. Y., Uejima, J. L., Reimers, J. M., Heng, L. J., Shaham, Y., Marinelli, M., and Wolf, M. E. (2008). Formation of accumbens GluR2 lacking AMPA receptors mediates incubation of cocaine craving. Nature 454, 118-121.

Costa, R. M., Cohen, D., and Nicolelis, M. A.L. (2004). Differential corticostriatal plasticity during fast and slow motor skill learning in mice. Curr. Biol. 14, 1124-1134.

DeLong, M., and Wichmann, T. (2010). Changing views of basal ganglia circuits and circuit disorders. Clin. EEG Neurosci. 41, 61-67.

Everitt, B. J., and Robbins, T. W. (2005). Neural systems of reinforcement for drug addiction: from actions to habits to compulsion. Nat. Neurosci. 8, 1481-1489.

Gerdeman, G. L., Partridge, J. G., Lupica, C. R., and Lovinger, D. M. (2003). It could be habit forming: drugs of abuse and striatal synaptic plasticity. Trends Neurosci. 26, 184-192.

Graybiel, A. M., Aosaki, T., Flaherty, A. W., and Kimura, M. (1994). The basal ganglia and adaptive motor control. Science 265, 1826-1831.

Haber, S. (2008). Parallel and integrative processing through the Basal Ganglia reward circuit: lessons from addiction. Biol. Psychiatry 64, 173-174.

Haracz, J. L., Tschanz, J. T., Wang, Z., White, I. M., and Rebec, G. V. (1993). Striatal single-unit responses to amphetamine and neuroleptics in freely moving rats. Neurosci. Biobehav. Rev. 17, 1-12.

Higgins, S. T., Budney, A. J., and Bickel, W. K. (1994). Applying behavioral concepts and principles to the treatment of cocaine dependence. Drug Alcohol Depend. 34, 87-97.

behavioral modification therapy for psychostimulant abuse (see Higgins et al., 1994, for review) may be useful for reversing some of the alterations in corticostriatal transmission occurring during the early phases of habitual drug taking.

\section{ACKNOWLEDGMENTS}

This work was supported by the Chicago Medical School at Rosalind Franklin University (Anthony R. West) and by National Institutes of Health Grant DA011261 (Heinz Steiner). We thank Joel Beverley for excellent technical assistance.

Hyman, S. E., and Malenka, R. C. (2001). Addiction and the brain: the neurobiology of compulsion and its persistence. Nat. Rev. Neurosci. 2 , 695-703.

Jog, M. S., Kubota, Y., Connolly, C. I., Hillegaart, V., and Graybiel, A. M. (1999). Building neural representations of habits. Science 286 1745-1749.

Kelley, A. E. (2004). Memory and addiction: shared neural circuitry and molecular mechanisms. Neuron 44 161-179.

Kourrich, S., Rothwell, P.E., Klug, J. R., and Thomas, M. J. (2007). Cocaine experience controls bidirectional synaptic plasticity in the nucleus accumbens. $J$. Neurosci. 27, 7921-7928.

Mahon, S., Deniau, J. M., and Charpier, S. (2004). Corticostriatal plasticity: life after the depression. Trends Neurosci. 27, 460-467.

Mallet, N., Le Moine, C., Charpier, S. and Gonon, F. (2005). Feedforward inhibition of projection neurons by fast-spiking GABA interneurons in the rat striatum in vivo. J. Neurosci. 25, 3857-3869.

Mogenson, G. J., Jones, D. L., and Yim, C. Y. (1980). From motivation to action functional interface between the limbic system and the motor system. Prog. Neurobiol. 14, 69-97.

Nestler, E. J. (2001). Molecular basis of long-term plasticity underlying addiction. Nat. Rev. Neurosci. 2, 119-128.

Nicola, S. M., Surmeier, J., and Malenka, R. C. (2000). Dopaminergic modulation of neuronal excitability in the striatum and nucleus accumbens. Annu. Rev. Neurosci. 23, 185-215.

Ondracek, J. M., Dec, A., Hoque, K. E., Lim, S. A., Rasouli, G., Indorkar, R. P., Linardakis, J., Klika, B., Mukherji, S. J., Burnazi, M., Threlfell, S., Sammut, S., and West, A. R. (2008). Feed-forward excitation of striatal neuron activity by frontal cortical activation of nitric oxide signaling in vivo. Eur. J. Neurosci. 27, 1739-1754.

Packard, M. G., and Knowlton, B. J. (2002). Learning and memory functions of the basal ganglia. Annu. Rev. Neurosci. 25, 563-593.
Paxinos, G., and Watson, C. (1998). The Rat Brain in Stereotaxic Coordinates. New York: Academic Press.

Pederson, C. L., Wolske, M., Peoples, L. L., and West, M. O. (1997). Firing rate dependent effect of cocaine on single neurons of the rat lateral striatum. Brain Res. 760, 261-265.

Picconi, B., Centonze, D., Håkansson, K., Bernardi, G., Greengard, P., Fisone, G. Cenci, M. A., and Calabresi, P. (2003). Loss of bidirectional striatal synaptic plasticity in L-DOPA-induced dyskinesia. Nat. Neurosci. 6, 501-506.

Rebec, G. V., White, I. M., and Puotz, J. K. (1997). Responses of neurons in dorsal striatum during amphetamine-induced focused stereotypy. Psychopharmacology 130, 343-351.

Reynolds, J. N., Hyland, B. I., and Wickens, J. R. (2001). A cellular mechanism of reward-related learning. Nature 413 , 67-70.

Reynolds, J. N., and Wickens, J. R. (2002). Dopamine-dependent plasticity of corticostriatal synapses. Neural. Netw. 15, 507-521.

Robbins, T. W., and Everitt, B. J. (1999). Drug addiction: bad habits add up. Nature 398, 567-570.

Sammut,S.,Dec,A.,Mitchell,D.,Linardakis, J., Ortiguela, M., and West,A. R. (2006). Phasic dopaminergic transmission Increases NO efflux in the rat dorsal striatum via a neuronal NOS and a dopamine $\mathrm{D}(1 / 5)$ receptor-dependent mechanism. Neuropsychopharmacology 31, 493-505.

Sammut, S., Threlfell, S., and West, A. R. (2010). Nitric oxide-soluble guanylyl cyclase signaling regulates corticostriatal transmission and short-term synaptic plasticity of striatal projection neurons recorded in vivo. Neuropharmacology 58, 624-631.

Schultz, W., Tremblay, L., and Hollerman, J. R. (2003). Changes in behavior-related neuronal activity in the striatum during learning. Trends Neurosci. 26, 321-328.

Steiner, H. (2010). "Psychostimulantinduced gene regulation in corticostriatal circuits," in Handbook of Basal Ganglia Structure and Function, eds H. Steiner and K. Y. 
Tseng (London: Academic Press/ Elsevier), 501-525.

Surmeier, D. J., Ding, J., Day, M., Wang, Z., and Shen, W. (2007). D1 and D2 dopamine-receptor modulation of striatal glutamatergic signaling in striatal medium spiny neurons. Trends Neurosci. 30, 228-235.

Threlfell, S., Sammut, S., Menniti, F. S., Schmidt, C. J., and West, A. R. (2009). Inhibition of phosphodiesterase 10A increases the responsiveness of striatal projection neurons to cortical stimulation. J. Pharmacol. Exp. Ther. 328, 785-795.

Tschanz, J. T., Haracz, J. L., Griffith, K. E., and Rebec, G. V. (1991). Bilateral cortical ablations attenuate amphetamine-induced excitations of neostriatal motor-related neurons in freely moving rats. Neurosci. Lett. 134, 127-130.

Unal, C. T., Beverley, J. A., Willuhn, I., and Steiner, H. (2009). Long-lasting dysregulation of gene expression in corticostriatal circuits after repeated cocaine treatment in adult rats: effects on zif 268 and homer 1a. Eur. J. Neurosci. 29, 1615-1626.

Ungless, M. A., Whistler, J. L., Malenka, R. C., and Bonci, A. (2001). Single cocaine exposure in vivo induces long-term potentiation in dopamine neurons. Nature 411, 583-587.
West, A. R., Floresco, S. B., Charara, A., Rosenkranz, J. A., and Grace, A. A. (2003). Electrophysiological interactions between striatal glutamatergic and dopaminergic systems. Ann. N. Y. Acad. Sci. 1003, 53-74.

West, A. R., and Grace, A. A. (2000). Striatal nitric oxide signaling regulates the neuronal activity of midbrain dopamine neurons in vivo. $J$. Neurophysiol. 83, 1796-1808.

West, A. R., and Grace, A. A. (2004). The nitric oxide-guanylyl cyclase signaling pathway modulates membrane activity states and electrophysiological properties of striatal medium spiny neurons recorded in vivo. J. Neurosci. 24, 1924-1935.

West, M. O., Carelli, R. M., Pomerantz, M., Cohen, S.M., Gardner,J.P., Chapin,J.K., and Woodward, D. J. (1990). A region in the dorsolateral striatum of the rat exhibiting single-unit correlations with specific locomotor limb movements. J. Neurophysiol. 64, 1233-1246.

West, M. O., Peoples, L. L., Michael, A. J., Chapin, J. K., and Woodward, D. J. (1997). Low-dose amphetamine elevates movement-related firing of rat striatal neurons. Brain Res. 745, 331-335.

White, F. J., and Kalivas, P. W. (1998). Neuroadaptations involved in amphetamine and cocaine addic- tion. Drug Alcohol Depend. 51, 141-153.

White, N. M. (1996). Addictive drugs as reinforcers: multiple partial actions on memory systems. Addiction 91 , 921-949.

White, N. M. (1997). Mnemonic functions of the basal ganglia. Curr. Opin. Neurobiol. 7, 164-169.

Willuhn, I., and Steiner, H. (2005). "Motor learning-related gene regulation in the striatum: effects of cocaine," in The Basal Ganglia VIII, eds J. P. Bolam, C. A. Ingham, and P. J. Magill (New York: Plenum Press), 197-207.

Willuhn, I., and Steiner, H. (2006). Motor-skill learning-associated gene regulation in the striatum: effects of cocaine. Neuropsychopharmacology 31 , 2669-2682.

Willuhn, I., and Steiner, H. (2008). Motorskill learning in a novel running-wheel task is dependent on D1 dopamine receptors in the striatum. Neuroscience 153, 249-258.

Willuhn, I., and Steiner, H. (2009). Skillmemory consolidation in the striatum: critical for late but not early long-term memory and stabilized by cocaine. Behav. Brain Res. 199, 103-107.

Willuhn, I., Sun, W., and Steiner, H. (2003). Topography of cocaine-induced gene regulation in the rat striatum: relationship to cortical inputs and role of behavioural context. Eur. J. Neurosci. 17, 1053-1066.

Wilson, C. J. (2004). "Basal ganglia," in The Synaptic Organization of the Brain, ed. G. Shepherd (Oxford: Oxford University Press), 361-414.

Conflict of Interest Statement: The authors declare that the research was conducted in the absence of any commercial or financial relationships that could be construed as a potential conflict of interest.

Received: 07 September 2010; accepted: 15 December 2010; published online: 29 December 2010.

Citation: Ondracek JM, Willuhn I, Steiner $H$ and West AR (2010) Interactions between procedural learning and cocaine exposure alter spontaneous and cortically evoked spike activity in the dorsal striatum. Front. Neurosci. 4:206. doi: 10.3389/ fnins.2010.00206

This article was submitted to Frontiers in Neuropharmacology, aspecialty of Frontiers in Neuroscience.

Copyright ( 2010 Ondracek, Willuhn, Steiner and West. This is an open-access article subject to an exclusive license agreement between the authors and the Frontiers Research Foundation, which permits unrestricted use, distribution, and reproduction in any medium, provided the original authors and source are credited. 